\title{
Comparison of Correlation Analysis and JointICA for Simultaneous EEG-fMRI recordings on Contour Integration Task
}

\author{
Bogdan Mijović, Bart Machilsen, Borbála Hunyadi, Maarten De Vos, Johan Wagemans, and Sabine Van Huffel
}

\begin{abstract}
Multimodal approaches to brain imaging are getting increasingly popular among the neuroscience comunity. One such multimodal approach is the combination of electroencephalography (EEG) and functional magnetic resonance imaging (fMRI). In this paper we demonstrate two EEGfMRI integration methods for contour integration task. First, we derrive the contour-selectivity measures from event related potential (ERP) and fMRI data, and explore the correlation between the two. In this way, we connect the spatial information from fMRI with the temporal information from ERPs. Thereafter, the results from this approach are compare to JointICA integration approach [5], [6], which aims at extracting spatiotemporal independent components, which are the combination of ERP and fMRI activations.
\end{abstract}

\section{INTRODUCTION}

Vision science aims to understand how the brain translates the pattern of light on the retina into a stable, coherent and meaningful picture of the outside world. An intermediate step in this process is to determine which parts of the retinal image belong together. Therefore, our research focuses on investigating the principles of contour integration, i.e. the grouping of local edges into global contours.

The involvement of both striate and extrastriate brain areas in contour integration has been observed in functional magnetic resonance imaging (fMRI) studies. It has been shown in [1], [2] that Gabor elements arranged to form a closed loop evoke stronger blood oxygenation level dependent (BOLD) response, than the Gabor elements that are randomly shown on the background. However, fMRI can only provide us with the spatial information, that is "where" the BOLD activation is present. It provides no temporal information on a subsecond time-scale. Therefore, brain-imaging techniques with a higher temporal resolution are required.

B. Mijović, B. Hunyadi and S. Van Huffel are with KU Leuven, Department of Electrical Engineering (ESAT-SCD), and iMinds Future Health Department, 3001 Leuven, Belgium, e-mail: bogdan.mijovic@esat.kuleuven.be.

B. Machilsen and J. Wagemans are with the Department of Psychology, division of Experimental Psychology, KU Lueven.

M. De Vos is with the Institute of Psychology, Universität Oldenburg, Department of Neuropsychology,

This research is supported by the Research Council KUL: IDO/05/010 (EEG-fMRI integration), GOA MaNet and PFV/10/002 (OPTEC); IUAP P719 (DYSCO, 20122017); the Flemish Government: FWO project G.0427.10N Integrated EEG-fMRI, IWT-TBM080658-MRI (EEG-fMRI) and iMinds. B. Mijović is supported by a PDM postdoctoral fellowship of KU Leuven, Belgium. B. Machilsen is a postdoctoral fellow of the Research Foundation-Flanders. M. De Vos is supported by an Alexander von Humboldt grant and J. Wagemans by long-term structural funding from the Flemish Government (METH/08/02).
For the purpose of this study, simultaneous EEG-fMRI recordings are used to retrieve fine spatio-temporal information. A potential measure which may provide information about the latency in which a given brain region processes information is the correlation between fMRI and ERP, in particular if a temporal dissociation between different regions can be found. Similar approach has been exploited in [3] to explore which of the three face-selective regions (FFA, fSTS and OFA) have responses that correlate with the event related potential (ERP) face-selective N170 component recorded from the $\mathrm{Cz}$ electrode.

Novel neurocognitive findings are discussed in [4]. In this study, we aim at comparing two different methods to extract spatio-temporal information from the recordings, a correlation based approach, as proposed in Sadeh et al., [3], and JointICA [5], [6]. The study also aims at showing the performance and highlighting the advantages and drawbacks of a data-driven approach compared to a correlation approach on contour integration data.

\section{METHODS}

\section{A. Participants}

15 participants (7 female and 8 male, aged 23-44, with a mean and standard deviation of respectively 27.4 and 5.2) with no history of neurological or cardiological disorders volunteered for this study. All participants reported normal or corrected-to-normal vision. Written informed consent was obtained in accordance with the KU Leuven Ethics Committee guidelines.

\section{B. Stimuli}

The task used in this work is explained in detail in [4]. Here we only provide a short description of the paradigm, which is important to understand the main conclusions of this particular study.

The employed stimuli were arrays of oriented and nonoverlapping Gabor elements, created with MATLAB and GERT, the Grouping Elements Rendering Toolbox [7]. The stimuli were presented centrally on a uniform grey screen with a randomized alternation between local+global contour (LG) and no contour (NC). NC stimuli were dense matrices of isolinear non-structure elements. In LG structure stimuli were dense matrices with curvilinear contour elements and isolinear non-structure elements. For more detailed explanation of this paradigm see [4], and for similar stimulus constructions, see [8]. 


\section{Experimental Procedure}

Each stimulus was presented for $200 \mathrm{~ms}$. The duration of the inter-trial interval was uniformly sampled between 2000 and $2400 \mathrm{~ms}$. A central fixation cross was shown during the inter-trial interval. The experiment was run using the Presentation software (Neurobehavioral Systems, Albany, CA, USA). A Barco projected the stimuli on a translucent screen attached to the bore of the scanner. Responses to the catch trials were registered using a response box.

\section{Localizers}

In addition to our task paradigm focusing on contour integration, fMRI data were acquired during four more runs especially intended to accurately define certain regions of interest (ROIs). This part is important in order to select the voxels, which are to be used further on in the correlation analysis. Two types of these so-called localizer experiments were performed thus allowing an accurate localization of the early visual areas (EVAs) V1 and V2 on the one hand, and lateral occipital complex (LOC) involved in object detection on the other hand. For more details see [4].

\section{E. Functional MRI}

1) fMRI acquisition: To acquire the fMRI images, a Philips 3T Intera whole-body scanner (Royal Philips Electronics, Amsterdam, the Netherlands) was used. The acquisition parameters differed between the experimental (related to contour integration) and the localizer runs. For each of the experimental runs, 155 echo-planar images (EPI) composed of 36 slices of $3 \times 3 \times 3 \mathrm{~mm}$ voxel size and $3 \mathrm{~mm}$ slice thickness were recorded with ascending slice order with 2 $\mathrm{s}$ repetition time (TR) and $29.8 \mathrm{~ms}$ echo time (TE). For the localizer runs, 110 images were acquired composed of 48 slices with $\mathrm{TR}=3 \mathrm{~s}$ and $\mathrm{TE}=29.8 \mathrm{~ms}$. In addition to the functional data, a full brain anatomical image was obtained with the magnetization prepared rapid gradient echo (MPRAGE) imaging sequence (182 coronal slices, $\mathrm{TE}=4.6$ $\mathrm{ms}, \mathrm{TR}=9.7 \mathrm{~s})$.

2) fMRI preprocessing: fMRI analysis was performed with the statistical parametric mapping software (SPM5, Wellcome Department of Cognitive Neurology, London, UK). The EPI time series were slice-time corrected, realigned, co-registered with anatomical images, normalized to a template and smoothed with a 6-mm FWHM Gaussian kernel. Next, the percentage signal change fMRI activation maps were retrieved via a general linear model (GLM) analysis with stick-functions based on the onset times of the different stimuli.

3) Definition of Regions of Interest: To define the regions of interest, first, the anatomical images of all participants were segmented and flat-maps were derrived using the Caret5 software (Van Essen Laboratory, Washington University, St. Louis, USA). Subsequently, the SPM-derrived activation maps (T-maps) from the object-localizer and meridianmapping runs were projected onto these flat-maps, and thresholded based on a p-value of 0.001 . The resulting overlays allowed deriving the LOC ROIs (for the objectlocalizer maps) and the ROIs in the early visual regions V1 and V2 (for the meridian-mapping maps).

4) Contour Selectivity Index: To make the correlation analysis meaningful, the selectivity index has to be defined, as proposed in [9]. Similar procedure is used in [3] to correlate face-selective voxels from fMRI with face-selective ERP repsonses.

For the purpose of our study, we define the contourselectivity index. This index is defined as normalized difference between the responses to contour and no contour stimuli as : Contour-NoContour (see [9]). In this way, the amplitudes for the fMRI response are kept between 0 and 1 (since all the responses were found to be positive).

\section{F. EEG Data}

1) EEG recording: The EEG data were collected from 62 standard scalp sites using the MR-compatible BrainAmp MR+ system (BrainProducts,Munich, Germany) with a sampling rate of $5 \mathrm{kHz}$. Two additional electrodes were placed below the left eye and on the left upper back to monitor eye blinks and the electrocardiogram (ECG), respectively. All 64 channels were recorded with $\mathrm{FCz}$ as reference and $\mathrm{Iz}$ as ground. Electrode impedances were kept below $10 \mathrm{k} \Omega$.

\section{G. EEG preprocessing}

The acquired EEG data were subjected to the standard preprocessing steps for the removal of scanner-related artifacts which arise from simultaneous EEG-fMRI acquisition [10]. These include gradient artifact, and balistocardiogram artifacts. Preprocessing as well as artifact removal were performed in the MATLAB (v 7.7; The MathWorks, Natick, MA, USA) environment with the EEGLAB 5.03 toolbox [11].

To extract task-related event-related potentials (ERPs), all available blocks per subject and per condition were merged together and data were segmented from $100 \mathrm{~ms}$ before until $500 \mathrm{~ms}$ after stimulus onset. Baseline correction was performed based on the $100 \mathrm{~ms}$ pre-stimulus interval and low quality trials were rejected by thresholding trials at 150 $\mu V$. Thereafter, an average ERP for each stimulus type was computed. The recording from the $\mathrm{Oz}$ electrode is used for this study

1) Contour Selectivity Index: Similar to the fMRI contour-selective index, for each subject normalized contourselectivity is computed as $\frac{\text { Contour-NoContour }}{\text { Contour }+ \text { NoContour }}$. Unlike the amplitude of the hemodynamic response that was always positive for both contour and no-contour stimuli for all subjects, the average ERP peaks derrived from the Oz electrode had opposite signs, which caused a deviant value that is larger than 1, whereas a ratio index of selectivity scores is only meaningful within the -1 to 1 range. Therefore a fixed value, which was slighly larger than the largest positive amplitude was subtracted from all the ERP's, which then results in all the normalized ERP values dropping inside -1 to 1 interval. This base-line correction method is proposed first in [9], and later used in [3]. 


\section{H. Data Analysis - Correlations}

In order to integrate EEG and fMRI, Sadeh et al., [3] proposed to correlate narrow time bins of a difference ERP waves (in their case responses to faces versus responses to houses) with the bold strength difference in certain regions of interest across subjects. In this way, inter subject variability is combined with the ERP time resolution in order to find "when" regions are active during the task. In [3] face processing is investigated. In this paper, we translate that approach to study contour integration.

The correlations are computed between the contourselectivity indexes derrived from ERP and fMRI data. This was done in order to couple the contour-selective spatial pattern obtained by fMRI imaging with the temporal patterns obtained from ERPs. Concerning the fMRI data, in each of the ROIs, the 30 most sensitive voxels are selected from the fMRI PSC map. The mean of these selected voxels is then used for the correlation with the ERP data. The ERP data, are divided in $4 \mathrm{~ms}$ windows, and for each of these windows the correlation between the ERP and fMRI contourselectivity indices is computed. This was done in the time interval of 0-350 $\mathrm{ms}$. For each $4 \mathrm{~ms}$ window, the p-value is computed without Bonferroni correction. In this way, the spatio-temporal dynamics are extracted.

\section{Data Analysis - JointICA}

In [4], the spatio-temporal information from the EEG and fMRI data acquired in this study is derrived using the JointICA approach [5], [6]. JointICA starts from the assumption that the amplitudes of the ERPs and the hemodynamic responses co-vary when they are generated in the same brain region. In this way, the fMRI activations for a particular stimulus are connected to the corresponding ERP peaks. Therefore, the independent components derrived from such an approach are a combination of temporal ERP wave and spatial fMRI map.

Additionally, the modified version of JointICA is proposed in [4], and applied to the contour integration task, used in this work. The results obtained with the modified JointICA approach are reused in this study to compare them with the results obtained with the correlation analysis as explained above. The modified JointICA constructs a mixed input data matrix for both paradigms, and performs the ICA decomposition jointly as shown in Eq. 1:

$$
\left[\begin{array}{ll}
\mathbf{X}_{L G}^{f M R} & \mathbf{X}_{L G}^{E E G} \\
\mathbf{X}_{N C}^{f M R I} & \mathbf{X}_{N C}^{E E G}
\end{array}\right]=\left[\begin{array}{l}
\mathbf{A}_{L G} \\
\mathbf{A}_{N C}
\end{array}\right] \cdot\left[\begin{array}{ll}
\mathbf{S}^{f M R I} & \mathbf{S}^{E E G}
\end{array}\right]
$$

\section{RESUlts}

The results from the correlation analysis are shown in Fig. 1. The correlations of the ERP difference wave with EVA (V1 and V2) and LOC areas differences are plotted in green, purple and black respectively. The dotted lines denote the time intervals in which the correlations are significant $(p<$ $0.05)$. The time interval of $0-350 \mathrm{~ms}$ is shown. The results imply that there is a significant correlation with LOC area

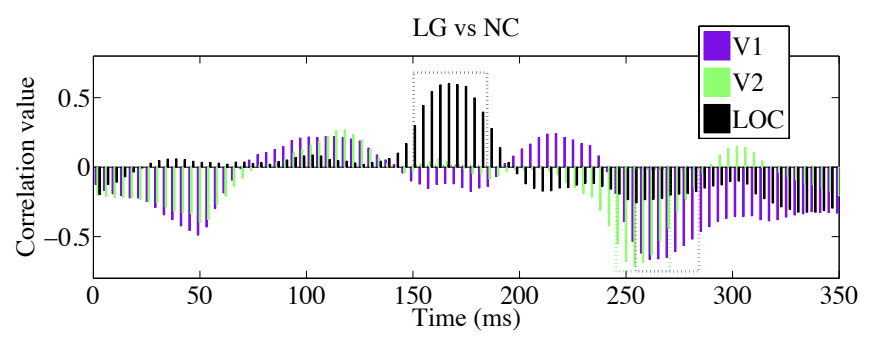

Fig. 1. The correlation between the activations in a specific ROI and the ERP values at specific time points. The bin-length in the ERPs is 4 milliseconds. Different ROIs are shown in different colors. The early visual area (EVA) ROI is shown in blue (V1) and green (V2), whereas the LOC ROI is shown in purple. The colors correspond to the ones in Fig. 2. The dotted lines denote the parts where the correlation is significant ( $\mathrm{p}$-value is smaller than 0.05).

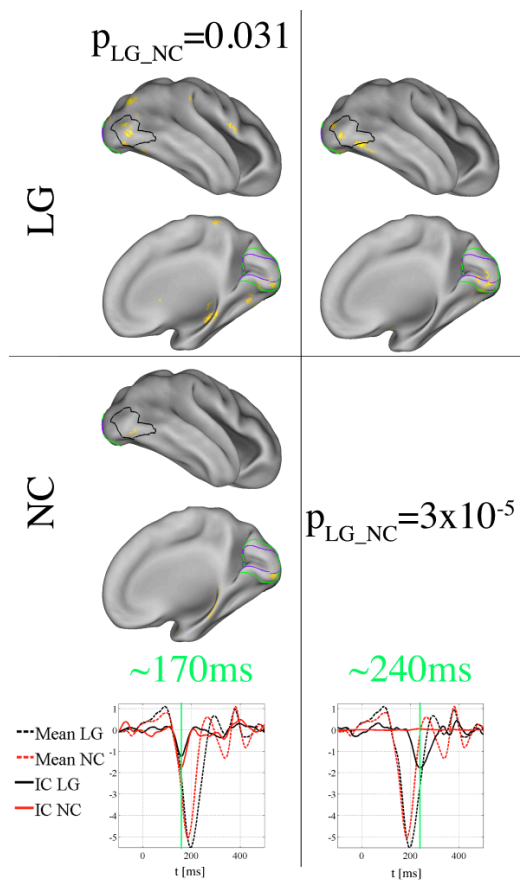

Fig. 2. Results of the modified JointICA analysis, performed jointly on the data from all three conditions (LG = local and global and $\mathrm{NC}=$ no contour). The upper panels show the spatial (fMRI) results from the condition-specific independent components, for (from top to bottom) the LG and NC conditions. We only show the components that show significant difference between the groups. The p-values denote the significance of the distribution difference across subjects (see [4]). The bottom panel presents the temporal (ERP) parts of the independent components, with different colors distinguishing between the different conditions. The ICs are plotted in solid line, whereas the grand average ERPs for all conditions are depicted by dashed lines.

around $170 \mathrm{~ms}$ and with EVA, first with V2 around $250 \mathrm{~ms}$, and later also with V1 around $270 \mathrm{~ms}$.

The results from the JointICA analysis are depicted in Fig. 2. Only the components which show significant differences for the two conditions (NC and LG) are plotted. Therefore, only two components are shown, and the p-values for the significance are given. The p-values, in this case, explains the significant difference in distribution across subject of the specific independent component (for more information, see [4]). Other components are not omitted. The colors of the 
LOC and EVA areas correspond to the colors from Fig. 1.

The top two panels show the fMRI parts of the independent components for the NC and LG conditions respectively. The bottom panel gives the ERP waves corresponding to the activations in the corresponding fMRI maps.

The results imply that the differences exist only around $170 \mathrm{~ms}$ and $240 \mathrm{~ms}$, which is in accordance with Fig. 1. Also, the fMRI maps show stronger activations in the LOC area for the LG condition around $170 \mathrm{~ms}$ compared to the NC condition, whereas the EVA activations do not differ. This also corresponds to the findings from Fig. 1. The component around $240 \mathrm{~ms}$ shows no ERP activity for the NC condition. For the LG condition the fMRI maps show mainly activations of the EVA area, but also some minor activation in LOC. This fact is also confirmed by our correlation analysis.

\section{DISCUSSION}

Integration of EEG and fMRI for brain imaging has been extensively used in recent years. However, no standard integration procedure has been established yet. In this study, therefore, we exploit two different integration approaches. The EEG-fMRI data acquired during a perceptual grouping experiment, were analyzed with correlation analysis, in a way explained in Methods section. These results are thereafter compared to the results from JointICA analysis, presented in [4] for further discussion.

In JointICA the variation across subjects is exploited to separate independent components, whereas the correlation analysis, also the strengths across subjects are comparted in narrow time bins.

Our results showed that the findings obtained from JointICA and correlation analysis mutually correspond. Both analyses show significant differences around $170 \mathrm{~ms}$ and 240-280 $\mathrm{ms}$, and the fMRI activations in LOC and EVA areas respectively. These results are also in line with the previous finding from the literature. Tanskanen et al., [12] found that responses to collinear contours embedded in a field of randomly-oriented Gabor elements began to differ from no-contour stimuli only after $130 \mathrm{~ms}$. Furthermore, Mathes et al., [13] found a contour-specific EEG response, starting about $150 \mathrm{~ms}$ after stimulus onset (see also [14]).

The correlation analysis shows positive correlation coefficient for contour selectivity indices corresponding to LOC and negative correlation coefficient for contour selectivity indeces corresponding to the EVA ROIs. Around the 170 $m s$, the NC condition shows stronger negative ERP peak than LG condition (see also Fig. 2). Therefore the ERP contour-selectivity index is positive, and the correlation is positive with the LOC. On the other hand, around $240 \mathrm{~ms}$, the LG condition shows stronger negative ERP peak than NC condition (see Fig. 2), providing negative contour-selectivity index, therefore yielding negative correlations with EVA areas.

The dissociation between V1 and V2 areas, as shown in Fig. 1 is not obtained with JointICA analsysis (Fig. 2). The reason for this may be in the fact that for the correlation analysis, all the V1 and V2 areas are subject specific, obtained through the Localizer analysis. On the other hand, JointICA cannot incorporate subject specific regions into ICA analysis. However, although indpendent from the localizer analysis, the JointICA fMRI activation indeed fall inside the ROIs derrived from the localizers. At the same time, JointICA is a fully data-driven method, and therefore, the localizer analysis is not necessary, whereas it is an absolute need in order to perform a meaningful correlation analysis.

Our research further showed that the JointICA analysis is very robust. Furthermore, ICASSO analysis of the jointICA technique showed the robustness of this approach [6]. On the other hand, the correlation analysis showed to be influenced by adding a single outlier subject. However, by following the procedures from [3] and [9], the correlation analysis also provides robust results.

This study provides the correlation analysis for EEG-fMRI data integration in a way proposed in [3], and described in the Methods section. The results are then compared to the JointICA results from [4] and the conclusions are derrived about the performances of JointICA and correlation analysis.

\section{REFERENCES}

[1] C. F. Altmann, H. H. Blthoff, and Z. Kourtzi, "Perceptual organization of local elements into global shapes in the human visual cortex," Current Biology, vol. 13, no. 4, pp. 342-349, 2003.

[2] Z. Kourtzi, A. Tolias, C. Altmann, M. Augath, and N. Logothetis, "Integration of local features into global shapes: Monkey and human fMRI studies," Neuron, vol. 37, no. 2, pp. 333-346, 2003.

[3] B. Sadeh, I. Podlipsky, A. Zhdanov, and G. Yovel, "Event-Related Potential and Functional MRI Measures of Face-Selectivity are Highly Correlated:A Simultaneous ERP-fMRI Investigation," Human Brain Mapping, vol. 31, no. 10, pp. 1490-1501, 2010.

[4] B. Mijović, M. De Vos, K. Vanderperren, B. Machilsen, S. Sunaert, S. Van Huffel, and J. Wagemans, "The dynamics of contour integration: A simultaneous eeg-fmri study," submitted paper, 2013.

[5] V. Calhoun, T. Adali, G. Pearlson, and K. Kiehl, "Neuronal chronometry of target detection: fusion of hemodynamic and event-related potential data," NeuroImage, vol. 30, pp. 544-553, 2006.

[6] B. Mijović, M. De Vos, K. Vanderperren, N. Novitskiy, B. Vanrumste, P. Stiers, B. Van den Bergh, J. Wagemans, L. Lagae, S. Sunaert, and S. Van Huffel, "The Why and How of JointICA: Results from a Visual Detection Task," NeuroImage, vol. 60, pp. 1171-1185, 2012.

[7] M. Demeyer and B. Machilsen, "Creating perceptual grouping displays with GERT," in Perception, 40; ECVP Abstract Supplement, 2011, p. 179.

[8] B. Machilsen, M. Pauwels, and J. Wagemans, "The role of vertical mirror symmetry in visual shape detection," Journal of Vision, vol. 9, no. 12:11, pp. 1-11, 2009.

[9] W. K. Simmons, P. S. F. Bellgowan, and A. Martin, "Measuring selectivity in fmri data," Nature Neuroscience, vol. 10, pp. 4-5, 2007.

[10] K. Vanderperren, M. De Vos, J. Ramautar, N. Novitskiy, M. Mennes, S. Assecondi, B. Vanrumste, P. Stiers, B. Van den Bergh, J. Wagemans, L. Lagae, S. Sunaert, and S. Van Huffel, "Removal of BCG artifacts from EEG recordings inside the MR scanner: a comparison of methodological and validation-related aspects," NeuroImage, vol. 50, no. 3 , pp. 920-934, 2010.

[11] A. Delorme and S. Makeig, "EEGLAB: an open source toolbox for analysis of single-trial EEG dynamics including independent component analysis," Journal of Neuroscience Methods, vol. 134, pp. 9-21, 2004.

[12] T. Tanskanen, J. Saarinen, L. Parkkonen, and R. Hari, "From local to global: Cortical dynamics of contour integration," Journal of Vision, vol. 8, no. 7:15, pp. 1-12, 2008.

[13] B. Mathes, D. Trenner, and M. Fahle, "The electrophysiological correlate of contour integration is modulated by task demands," Brain Research, vol. 1114, no. 1, pp. 98-112, 2006.

[14] B. Machilsen and J. Wagemans, "Integration of contour and surface information in shape detection," Vision Research, vol. 51, no. 1, pp. 179-186, 2011. 